Research paper

\title{
Distinct gene networks drive differential response to abrupt or gradual water deficit in potato
}

\author{
Alfredo Ambrosone ${ }^{\mathrm{a}, 1}$, Giorgia Batelli ${ }^{\mathrm{a}}$, Hamed Bostan ${ }^{\mathrm{b}}$, Nunzio D'Agostino ${ }^{\mathrm{c}}$, Maria Luisa Chiusano ${ }^{\mathrm{b}}$, \\ Gaetano Perrotta ${ }^{\mathrm{d}}$, Antonietta Leone ${ }^{\mathrm{e}}$, Stefania Grillo ${ }^{\mathrm{a}}$, Antonello Costa ${ }^{\mathrm{a}, *}$ \\ a National Research Council of Italy, Institute of Biosciences and BioResources, Research Division Portici (CNR-IBBR), Portici (NA), Italy \\ b Department of Agricultural Sciences, University of Naples "Federico II", Portici (NA), Italy \\ c Consiglio per la ricerca in agricoltura e l'analisi dell'economia agraria, Centro di ricerca per l'orticoltura (CREA-ORT), Pontecagnano Faiano (SA), Italy \\ d Italian National Agency for New Technologies, Energy and Sustainable Economic Development (ENEA), TRISAIA Research Center, Rotondella, MT, Italy \\ e Department of Pharmacy, University of Salerno, Fisciano SA, Italy
}

\section{A R T I C L E I N F O}

Article history:

Received 24 June 2016

Received in revised form 8 October 2016

Accepted 18 October 2016

Available online $\mathrm{xxxx}$

\section{Keywords:}

Plant stress and adaptation

Potato

Solanum tuberosum

Water stress

\begin{abstract}
A B S T R A C T
Water-limiting conditions affect dramatically plant growth and development and, ultimately, yield of potato plants (Solanum tuberosum L.). Therefore, understanding the mechanisms underlying the response to water deficit is of paramount interest to obtain drought tolerant potato varieties. Herein, potato $10 \mathrm{~K}$ cDNA array slides were used to profile transcriptomic changes of two potato cell populations under abrupt (shocked cells) or gradual exposure (adapted cells) to polyethylene glycol (PEG)-mediated water stress. Data analysis identified $>1000$ differentially expressed genes (DEGs) in our experimental conditions. Noteworthy, our microarray study also suggests that distinct gene networks underlie the cellular response to shock or gradual water stress. On the basis of our experimental findings, it is possible to speculate that DEGs identified in shocked cells participate in early protective and sensing mechanisms to environmental insults, while the genes whose expression was modulated in adapted cells are directly involved in the acquisition of a new cellular homeostasis to cope with water stress conditions.

To validate microarray data obtained for potato cells, the expression analysis of 21 selected genes of interest was performed by Real-Time Quantitative Reverse Transcription PCR (qRT-PCR). Intriguingly, the expression levels of these transcripts in 4-week old potato plants exposed to long-term water-deficit. qRT-PCR analysis showed that several genes were regulated similarly in potato cells cultures and tissues exposed to drought, thus confirming the efficacy of our simple experimental system to capture important genes involved in osmotic stress response. Highlighting the differences in gene expression between shock-like and adaptive response, our findings could contribute to the discussion on the biological function of distinct gene networks involved in the response to abrupt and gradual adaptation to water deficit.
\end{abstract}

(C) 2016 Elsevier B.V. All rights reserved.

\section{Introduction}

Water limiting conditions drastically affect plant growth and production. In particular, water deficit, which causes also osmotic stress, severely impairs plant development through the imposition of molecular and physiological changes that, in case of severe and durable water

Abbreviations: PEG, polyethylene glycol; DEG, differentially expressed gene; qRT-PCR Real-Time Quantitative Reverse Transcription PCR; TIGR, The Institute for Genomics Research; LOWESS, locally weighted linear regression; PGSC, potato genome sequencing consortium; EST, expressed sequence tag; ABA, abscisic acid.

* Corresponding author.

E-mail address: antcosta@unina.it (A. Costa).

${ }^{1}$ Present Address: Instituto de Ciencias de Materiales de Aragón (ICMA), Universidad de Zaragoza-CSIC, 50009 Zaragoza, Spain. deficit, lead to disorganization of cell membranes, metabolic toxicity and inhibition of photosynthesis (Zhu, 2001). However, even non tolerant plants respond and might adapt to drought by modulating the expression of several genes (Seki et al., 2003; Shinozaki et al., 2003; Urano et al., 2010; Yamaguchi-Shinozaki and Shinozaki, 2005). Usually, stress-related genes govern important cell mechanisms such as protein metabolism, transcriptional and post-transcriptional regulation, signalling and stress perception, ion homeostasis, detoxification and damage repair (Ambrosone et al., 2012; Bray, 2002; Rensink et al., 2005; Shinozaki et al., 2003). However, the survival or death of plants experiencing water deficit is known to depend not only on the plant genetic background, developmental stage and morphology, but also on the severity and duration of the stress event (Leone et al., 1994a). Therefore, while gradual and continuous imposition of water deficit can produce metabolic and molecular modifications that allow a new 
cellular homeostasis and active growth even in not optimal environmental conditions, a short exposure to severe water stress may activate genes involved in the prevention/repair of cellular damage, as well as genes involved in the stress perception/signalling function (Ambrosone et al., 2011; Leone et al., 1994b).

Many varieties of $S$. tuberosum, the most commonly consumed potato, are very sensitive to water-limiting conditions, which reduce marketable yield (Deblonde and Ledent, 2001; Weisz et al., 1994). Potato is the most important non-grain food crop, and given the increased occurrence of drought and its severity, great efforts need be addressed to understand genetic mechanisms controlling potato plant adaptation to water deficit. In that respect, recently published outcomes from the potato genome sequencing project have provided important genomic information allowing dissecting complex genetic traits of agronomical interest (The Potato Genome Sequencing Consortium, 2011).

Over the past two decades, we deeply investigated the physiological and molecular responses of potato cell cultures exposed to osmotic stresses, demonstrating that they respond in different ways if exposed abruptly or gradually to water deficit elicited by PEG addition into the culture medium (Leone et al., 1994a). Our previous studies indicated that water stress, when imposed gradually to potato cells, is compatible with active cellular growth, primarily due to restored normal protein synthesis (Leone et al., 1994b), proline and polyamine accumulation (Scaramagli et al., 2000) and changes in membrane composition and fluidity (Leone et al., 1996).

Although one can argue that a cellular system may not fully represent the complex in vivo plant behaviour to a changeable environment, investigating plant stress response at cellular level allows to identify genes mostly involved in cell stress perception and transduction, as well as in controlling and maintaining cellular homeostasis. Worth of note, several lines of evidence indicate that the experimental conditions used to impose plant water stress frequently do not reflect what really happens in soil. For instance, sudden exposure to severe water stress occur rarely in the field, while more often plants need to adapt to natural fluctuation of environmental conditions which make the physiological response much more complex than a simple experimental binary condition (stress vs control). Overall, this suggests that transcriptional changes conferring tolerance to severe water stress may not sustain plant growth under gradual adaptation to water limiting conditions (Claeys and Inzé, 2013; Claeys et al., 2014; Clauw et al., 2015). As consequence, the comprehension of these distinct dynamics is really appealing for genetic improvement programs conceived to select droughtresistant varieties (Blum, 2005).

With this respect, comparative transcription profiles of the response to abrupt or gradual water stress conditions may provide additional information about genes regulated in severe water stress that might belong to early responsive genes, providing initial protection and amplification of primary osmotic/water stress signals, and about genes whose expression is changed during gradual adaptation that might be involved in tolerance to stress conditions.

Because of the complexity of the overall plant response to drought stress, in recent years global transcriptome analyses have replaced the gene-by-gene approaches in different plant species to identify genes whose expression is regulated in response to water stress (Gullì et al., 2015; Iovieno et al., 2016; Kreps et al., 2002; Li et al., 2016; Roche et al., 2008; Seki et al., 2002; Xue et al., 2008) or to other abiotic stresses since it allows the simultaneous analysis of a great number of genes (Legay et al., 2009).

This study was aimed to capture and compare transcriptional profile changes in potato cells exposed to abrupt (shock) or gradual imposition (adaptation) to water stress by using a potato $10 \mathrm{~K}$ cDNA array slides containing approximately 10,000 potato cDNA clones. By exploiting an alternative route towards the identification of water stress responsive genes, we provided evidences that distinct gene networks are regulated in potato cell exposed to abrupt or gradual adaptation to water stress. Furthermore, to verify whether genes involved in the response of the whole plant to stress can be identified by an in vitro simplified cellular system, a selected number of stress responsive genes in potato cells was monitored in potato plants subjected to a mild drought-stress treatment. In conclusion, we demonstrated that potato cells respond to water deficit according to the intensity and the duration of water limiting conditions providing unique molecular signatures for potato stress adaptation in vitro and in vivo.

\section{Materials and methods}

\subsection{Cell cultures, plant growth and stress treatments}

Potato cell cultures were obtained from leaf callus of potato plants and subcultured every 7 days. Cells were maintained in a modified Murashige and Skoog medium in a rotary shaker at $28{ }^{\circ} \mathrm{C}$ in the dark as described in Leone et al., 1994a. Gradual acclimation to low water potential was achieved by transferring cells to nutrient medium containing increasing concentrations of PEG 8000 (5, 10, 15 and 20\% w/v). Cells were maintained at each PEG concentration for at least 2 subculture cycles and then for 45 subculture cycles at 20\% PEG. For shock conditions, control cells were transferred and maintained for $24 \mathrm{~h}$ into a medium containing $20 \%$ PEG.

Four-week-old potato plants were grown in greenhouse in pots containing peat:perlite $(1: 1)$ soil and subject to water stress for seven days by withholding water. Control potato plants of the same developmental stage were maintained in condition of regular water regime (daily watered). Stomatal resistance was measured daily during a seven-day treatment with a leaf porometer (AP4, Delta-T Devices). In each plant, measurements were carried out on basal, middle stem and apical leaves.

\subsection{RNA isolation}

Total RNAs were extracted from at least three biological replicates of control cells, cells subjected to PEG treatments and plant tissues (500 mg fresh weight) by the guanidinium isothiocyanate method according to Chomeczynski and Sacchi, 1987. The concentration of RNA samples was determined through spectrophotometric analysis. The extraction purity (absence of any contaminants, such as proteins, polysaccharides and phenol) was estimated by measurement the absorbance ratio OD260/280. The samples were considered for further analysis when the ratio A OD260/A OD280 had a value between 1.7 and 2.0. The RNA samples were examined by electrophoresis on agarose gel at $1,2 \%$ to check the absence of any degradation and contamination of residual genomic DNA. Total cell RNA (50 mg) from control, treated cells and plant tissues were incubated for $30 \mathrm{~min}$ at $37^{\circ} \mathrm{C}$ with 20 units of RNAse-free DNase I (Gibco BRL, Life Tecnologies, USA). After extraction with phenol/chloroform (3:1) and ethanol precipitation in the presence of $0.3 \mathrm{M}$ sodium acetate, the RNA was redissolved in $20 \mu \mathrm{L}$ of diethyl pyrocarbonate-treated water:

\subsection{Preparation of labelled probes and array hybridisation}

The potato $10 \mathrm{k}$ cDNA array version 3 provided by TIGR (The Institute for Genomics Research) was employed in the present study. Preparation of labelled probes, microarray hybridisation and data analysis was carried out in the TRISAIA Research Center (ENEA). To minimize the cyanine incorporation bias, we adopted the "dyeswap" hybridization (Dobbin et al., 2003) for each combination of probes (stressed vs control cells, adapted vs control cells). At least three technical and two biological replicates were carried out per hybridization.

RNA labelling was achieved using direct-labelling methods. For each direct-labelling reaction, $40 \mu \mathrm{g}$ of DNA-free total RNA were reverse transcribed using SuperScript II reverse transcriptase (Invitrogen) and oligodT primer in the presence of Cy3- or Cy5-labelled dUTP. Unincorporated 
nucleotides were eliminated using GFX-PCR columns (Amersham Biosciences) following manufacturer's specifications. Purified Cy3and Cy5-labelled cDNAs were dried in a speed-vac and resuspended in hybridization buffer (Amersham Bioscience) supplemented with $50 \%$ formamide, dispensed over the microarray glass slide (already pre-hybridized in $50 \%$ formamide buffer at $42{ }^{\circ} \mathrm{C}$ for $1 \mathrm{~h}$ and rinsed briefly in sterile water) and incubated at $42{ }^{\circ} \mathrm{C}$ overnight in agitation. Slides were washed in decreasing SSC concentrations $(2 \times-0.1 \times)$ and $0.1 \% \mathrm{SDS}$ at $42{ }^{\circ} \mathrm{C}$ and room temperature, respectively. The last wash was carried out in $0.1 \times$ SSC at room temperature. The fluorescence of individual hybridized spots was measured using the ScanArray Lite (Perkin-Elmer) and the resulting Cy3 and Cy5 images were analyzed with the software ScanArray Express (Perkin-Elmer) in order to measure the Сy3/Cy5 spot intensities.

\subsection{Microarray data analysis}

Local background was subtracted from the fluorescence value of each spot. Spots covered by dust particles or characterized by a false intensity due to a high background, missing spots and spots having low signal intensity were flagged and removed from the subsequent analysis.

Raw values were then normalized with the locally weighted linear regression (LOWESS) method using the $20 \%$ of data for smoothing (Cleveland and Devlin, 1988).

Transcript changes were expressed as the ratio of intensities between stressed $v s$ control RNAs and adapted vs control RNAs. The mean values of the data sets produced by dye-reversal hybridisation were considered for analysis. Changes in the average signal intensity exceeding a two-fold or higher difference were declared significant only when the corresponding coefficient of variation was $<50 \%$ and the average intensity ratio differed from the threshold levels (2.0 and 0.5 for up- and down-regulation, respectively) by a value equal or higher than the standard deviation.

\subsection{Bioinformatic analysis}

Functional assignment of the differentially expressed/suppressed transcripts was performed by blasting the probe/EST sequences versus the reviewed SwissProt/UniprotKB database downloaded on 2014 using NCBI Blast (Tatusova and Madden, 1999) with an evalue $10 \mathrm{E}-3$. The first five best hits were collected for further functional investigations.

To identify the genomic locus of each transcript on the reference genome sequence, the cDNA sequence of the differentially expressed/ suppressed transcripts was aligned versus the Potato reference genome sequence (PGSC_DM version 4.03 downloaded from SpudDB (Hirsch et al., 2014) using GenomeThreader, a gene prediction software (Gremme et al., 2005) considering identity and coverage of 0.90 and 0.80 respectively. The predicted loci were then intersected with the PGSC_DM gene annotation version 4.03, downloaded from SpudDB (Hirsch et al., 2014) to identify the corresponding gene models from the official gene annotation.

\subsection{Validation of expression data}

Gene expression data were validated by qRT-PCR. The firststrand cDNA synthesis was carried out with the SuperScript II Retrotranscriptase (Invitrogen) and oligo dTs, according to the manufacturer's instructions. PCR reactions were run in a $25 \mu \mathrm{L}$ volume, containing $2 \times$ Platinum SYBR green qPCR SuperMix-UDG with ROX, serial cDNA dilutions and $0.5 \mu \mathrm{M}$ of each primer. cDNAs were amplified using GeneAmp PCR System 9700 (Applied Biosystems) as follows: an initial denaturation for $10 \mathrm{~min}$ at $94{ }^{\circ} \mathrm{C}$, followed by 40 cycles at $94{ }^{\circ} \mathrm{C}$ for $15 \mathrm{~s}, 59{ }^{\circ} \mathrm{C}$ for $30 \mathrm{~s}$, and $72{ }^{\circ} \mathrm{C}$ for $30 \mathrm{~s}$, and a 20 min gradient from $60{ }^{\circ} \mathrm{C}$ to $90{ }^{\circ} \mathrm{C}$ to obtain a melting curve. Specific primers for each gene were designed using Primer3 software (http://bioinfo.ut.ee/ primer3-0.4.0/) and are listed in Table S1.

All experiments were carried out using three biological and three technical replicates per experimental point. According to our previous gene expression analysis (Costa et al., 2010; Ambrosone et al., 2011), we employed the ribosomal 18S RNA as housekeeping gene in potato cells, while the elongation factor 1 -alpha (ef- $1 \alpha$ ) was employed as internal calibrator in potato plants. Relative expression data were calculated by using the delta-delta $\mathrm{Ct}\left(2^{\Delta \Delta \mathrm{CT}}\right)$ method (Livak and Schmittgen, 2001).

\section{Results}

\subsection{Potato transcriptome in response to water stress}

In order to dissect global gene expression modifications occurring during gradual plant adaptation with respect to abrupt water stress, large-scale expression analyses were conducted by microarray technology in three potato cell populations (Fig. S1): i) cell cultures maintained in optimal growth conditions (control); ii) cell cultures, exposed abruptly to hyperosmotic medium containing PEG $(20 \% \mathrm{w} / \mathrm{v})$ for $24 \mathrm{~h}$ (shocked cells); iii) cell suspensions cultured under long-term water stress conditions (adapted cells), obtained by gradual addition of PEG moieties over a 60 days subculturing period, until reaching 20\% PEG in the culture medium, as described in Leone et al., 1994a. Noteworthy, according to our previous reports, gradually water-stressed potato cells were able to sustain active proliferation, while cell growth was dramatically impaired in PEG-shocked cells with respect to untreated cells (Fig. S2).

Potato $10 \mathrm{~K}$ microarray slides containing approximately 10,000 ESTs from cDNA libraries of different tissues and species of potato, were used to profile global gene expression changes occurring in the different potato cell populations. Overall, 1045 genes from adapted and shocked potato cells exhibited a significant altered expression profile (overexpression or repression) compared to control cells. Among these, 130 genes were overexpressed in shocked cells (shocked up, Table S2) and 333 genes overexpressed in adapted cells (adapted up, Table S3), while 174 genes were down-regulated in response to shock conditions (shocked down, Table S4) and 408 down-regulated during cell adaptation (adapted down, Table S5).

In order to gain functional information, differentially expressed sequences were annotated by determining their closest matches into protein databases (SwissProt/UniprotKB) by BLAST analyses. Besides closest functional description reported by BLAST for each query, S2-S5 tables also provide details about fold changes (treated vs untreated potato cells), EST name, clone name and functional classification according to the FunCat catalog (Ruepp et al., 2004).

To provide the corresponding potato gene identifier (IDs), differentially expressed sequences were aligned along the potato reference genome. In addition, overlapping of the predicted genomic locus for each of the query sequences with the official gene annotation provided by Potato Genome Sequencing Consortium (PGSC) and all the other information concerning the genomic mapping of each sequence are included into the supplementary materials (potato dataset and Table S6). Small discrepancies in gene annotations have been observed between BLAST analysis and potato genome alignment.

Functional categories distributions of DEGs identified in the different potato cell populations are reported in Fig. 1, A-D. A significant percentage, from $31 \%$ to $46 \%$, of genes were classified as "experimentally uncharacterized proteins", a heterogeneous category including unknown sequences and similar to hypothetical proteins and uncharacterized genes.

Noteworthy, the category of transcription factors (TFs) was one of the functional classes most represented among the up-regulated transcripts. In particular, in shock conditions were significantly up-regulated at least 10 genes involved in transcription regulation, including transcription 
A

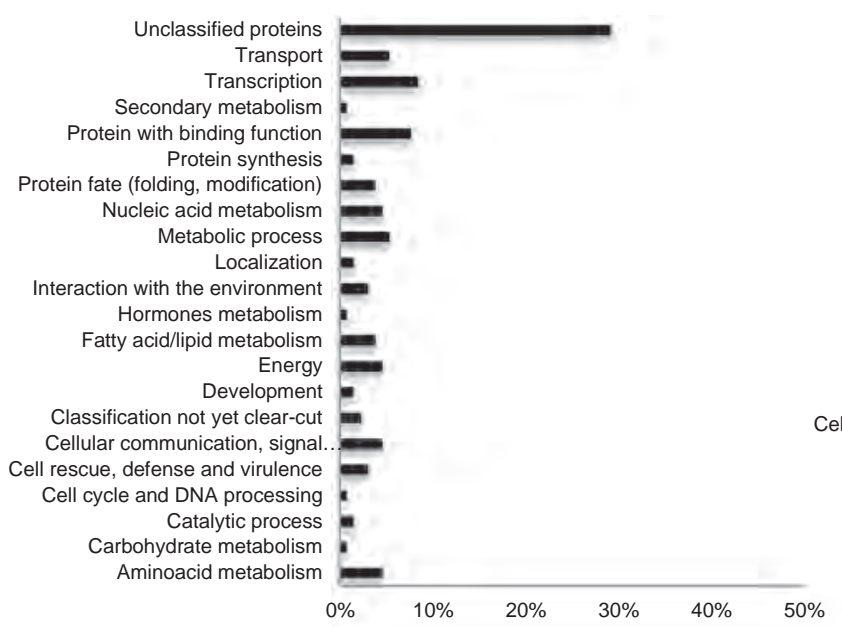

C

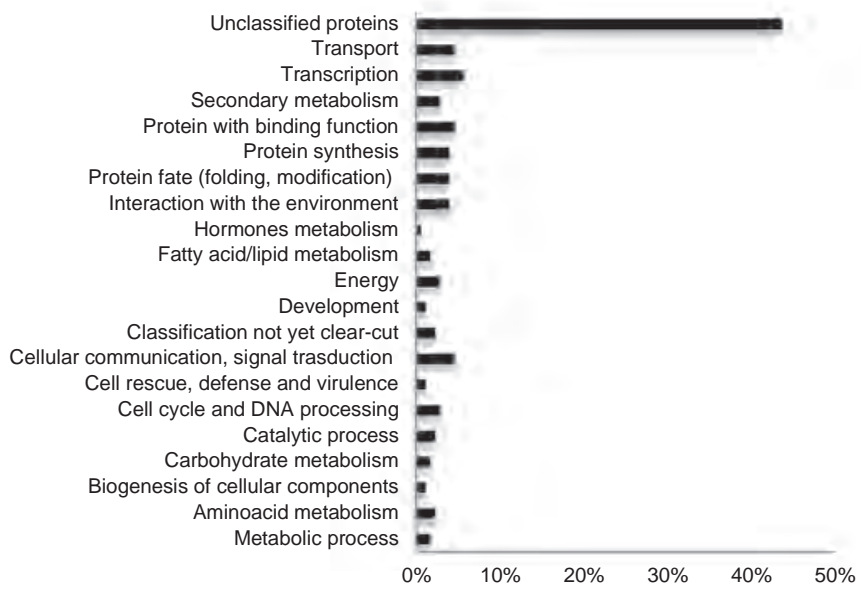

B Adapted up

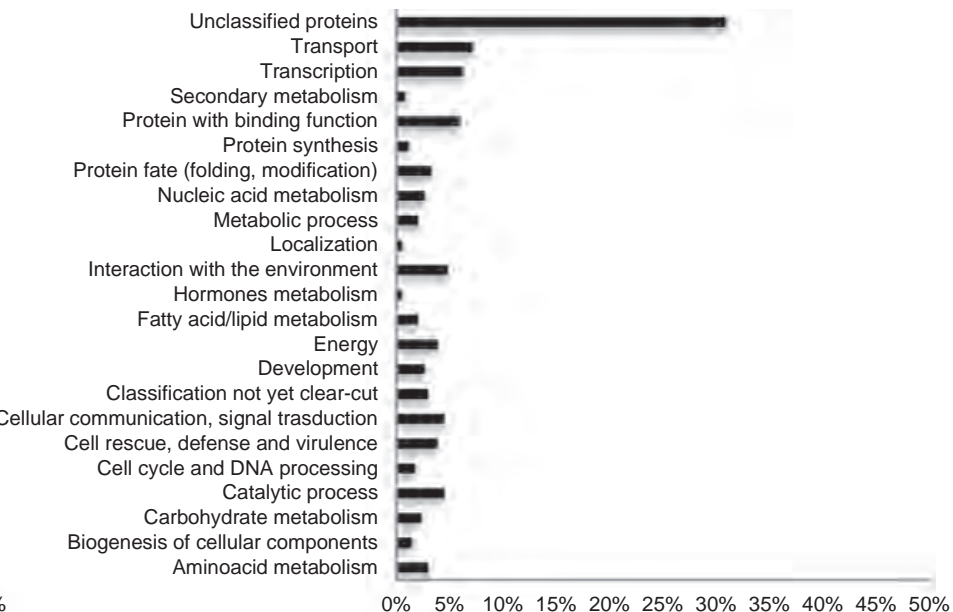

D Adapted down

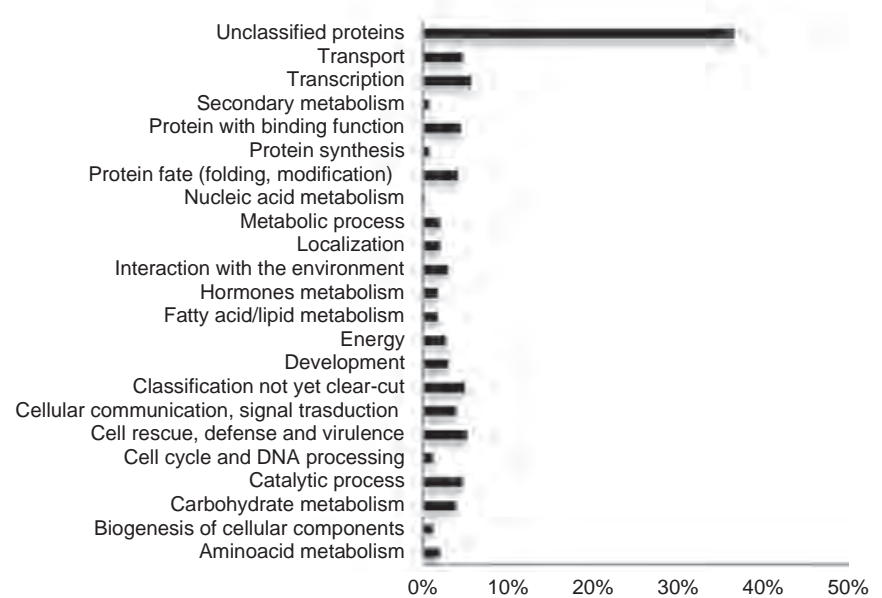

Fig. 1. Functional category distribution of differentially expressed genes: up regulated in shocked (A) and adapted (B) potato cells; down regulated in shocked (C) and adapted potato cells (D).

factors costans-like1 (COL1 BQ511758), gigantea (BQ510464), scarecrow (SCL1, BQ510154) and a transcription factor of the MYB family (BQ514240). The expression of TFs such as heat shock transcription factor A6B (HSFA6B, BQ511608), heat shock transcription factor B4 (AT-HSFB4, BQ505416), and zinc finger transcription factors (BQ120740) was suppressed in shocked cell populations. Among the transcription factors coding genes overexpressed during adaptation, auxin response factor 22 (arf22, BQ112621), G-BOX binding factor 3 ( $g b f 3, \mathrm{BQ} 514015$ ), sensitive to proton rhizotoxicity 1 (stop1, BQ507958), tiny (BQ115125) and wrky40 (BQ516587), which showed a higher induction levels ( $>25$-fold up-regulated), were identified.

Under shock conditions, many transcripts involved in protein synthesis were repressed, including genes coding for ribosomal proteins such as 40S ribosomal protein S3 (RPS3C, BQ509785), 60S acidic ribosomal protein P3 (RPP3B, BQ117876), 60S ribosomal protein L4/L1 (RPL4D, BQ119025), 60S ribosomal protein L10 A (RPL10aB, BQ115658). A vast repertoire of genes already known to be involved in stress response was differentially regulated in both adapted and shocked cell populations. In more details, genes coding for a glutathione S-transferase
(AtGSTU8, BQ510856) and an early light-induced protein (BQ519197) were induced exclusively in cells exposed to abrupt water deficit.

In adapted cell cultures, several stress-responsive genes including early-responsive to dehydration 8 (HSP81-2, BQ118343), Abscisic stress-ripening protein 2 (ASR2, BQ512572), salt tolerance homolog 2 (STH2, BQ511115), propyzamide-hypersensitive 1 (PHS1, BQ509645), osmotin-like protein OSML13 (BQ115133) and abscisic acid receptor PYL6 (BQ516958) were overexpressed; genes typically involved in the response to biotic stress, including transcripts coding for proteins labelled as "disease resistance" (BQ513155, BQ518444, BQ512657, BQ518456) protease inhibitors (BQ515885) and chitinase (BQ518165), were also induced.

From global data analysis, only 9 genes were commonly down-regulated. Namely, genes encoding an alanine aminotransferase (ALAAT2, BQ116186), cysteine synthase D1 (CYSD1, BQ510307), a DNA translocase (BQ519349), a putative pterin-4-alpha-carbinolamine dehydratase (BQ112737), a Glutathione S-transferase (BQ512747) and 4 unknown transcripts (BQ117767, BQ509807, BQ511641, BQ512797) were all commonly repressed in both cell populations compared to control cells. Taken together these data demonstrate that potato cells modulate divergent 
multiple pathways according to distinct mode of water stress experienced.

\subsection{Validation of microarray data and study of gene expression in $S$. tuberosum cells}

In order to validate microarray results, we investigated by Real-Time Quantitative Reverse Transcription PCR (qRT-PCR) the expression levels of 21 potato genes, randomly selected from the list of differentially expressed transcripts in shocked and adapted potato cells (Table S1).

For the amplification of each transcript, specific primers were designed in the EST sequences spotted on the slide. The expression data were obtained from at least 3 independent biological experiments with three technical replicates each. Overall, qRT-PCR analyses of selected gene confirmed microarray data patterns, according to the expression outcomes listed in Fig. 2. In particular, genes coding for a defective Kernel 1 (DEK1, BQ516930), a RNA polymerase II subunit (RBP10, BQ111440) and an unknown protein (BQ517616) showed the highest expression increases in shocked cells compared to control. Interestingly, a sucrose transporter showed a consistent specific induction in cells adapted to water stress. Conversely, many genes such as sucrose synthase, catalase, $A B C$ transporter, a F-box protein and an ion channel (cation exchanger 2 ) were induced exclusively in response to shock conditions. The $\mathrm{qRT}-\mathrm{PCR}$ analysis showed that some genes are repressed in both cell populations ( $a t g b 2$, a $40 \mathrm{~S}$ ribosomal protein and a protein containing a rhodanese-domain). Finally, a GDSL lipase was slightly overexpressed in adapted cells, but had an unmodified expression profile in cells subjected to abrupt water deficit.

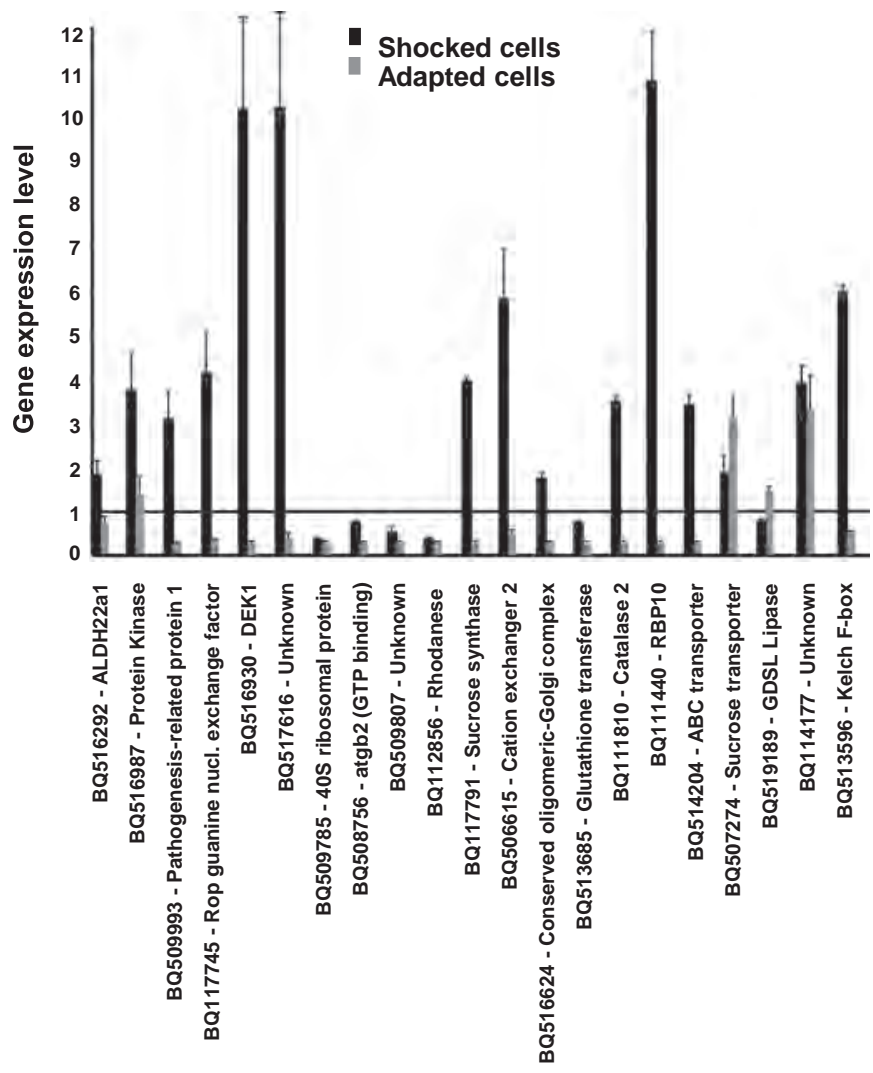

Fig. 2. Quantitative RT-PCR of representative genes identified in microarray analysis. Black bars indicate the expression levels of differentially expressed genes identified in shocked cells, while grey bars show genes identified in adapted cells. All the genes confirmed the same expression patterns observed in microarray analysis. Data are means \pm SD of three independent experiments.
3.3. From cells to plants: Expression profiles of water stress-responsive genes

Further experiments were carried out to uncover the role of the identified genes in in vivo plant response to water deficit. The expression of 10 representative upregulated transcripts, randomly selected from the DEGs list of shocked and adapted cells, was investigated by quantitative qRT-PCR analysis in four-week-old potato plants (cv. Desirée) subjected to water withdrawal for 7 days. The increased stomatal resistance, measured at the end of treatment, confirmed the real intensity of the stress imposed (Fig. S3). As shown in Fig. 3A, 9 out of 10 genes showed in potato leaves the same expression pattern as observed in cell cultures. In more details, protein kinase (BQ516987), sucrose synthase (BQ117791) and a cation exchanger (BQ506615) transcripts were highly overexpressed in leaves, respectively showing 41,25 and 39-fold changes with respect to leaves of plants grown in control condition.

In addition, genes encoding a pathogenesis-related prot 1, a catalase, a RNA polymerase subunits (RBP10), an oligomeric Golgi complex subunit and an unknown gene, were markedly induced in presence of water deficit. Conversely, the EST BQ514204 coding for an ABC transporter was repressed in leaves of potato grown under low water availability.

Surprisingly, gene expression analysis in roots of water stressed potato plants revealed that only two genes, coding for a protein kinase (BQ516987) and a cation exchanger 2 (BQ506615), were highly induced upon water deficit, while the other selected transcripts were either down-regulated or did not show significant modifications in their expression levels (Fig. 3B).

\section{Discussion}

Osmotic stress induces deep alterations of plant biochemical and physiological processes with obvious and well known consequences on crop productivity (Araus et al., 2002). The activation of stress-responsive mechanisms depend strictly on the duration and intensity of stress as well as on plant capability to trigger cellular responses in short and long-term and consequently to limit damage and preserve the cell structures (e.g. in shock conditions) and to restore a new homeostatic balance (e.g. in adaptive responses) (Leone et al., 1999).

Understanding how environmental factors tune specific gene networks is of utmost importance in the modern agriculture, not merely based on the optimization of culture conditions, sometimes impossible or economically disadvantageous, but rather on the ability to modulate the whole gene network profile in order to guarantee plant production, even in unfavourable conditions.

Here, we used potato cell cultures subjected to different water stress conditions, namely shock or adaptation, with the aim to elucidate the molecular basis of plant response to water stress, capturing genes and fundamental gene functions involved in these processes.

Cell cultures are valuable tools to elucidate the complexity of the mechanisms of transduction of exogenous signals, allowing focusing on the biochemistry and cell physiology underlying the maintenance of homeostasis under stress conditions (Grillo et al., 1996; Takahashi et al., 2004). Although the experimental system used represents a simplification of physiological mechanisms adopted by plants, it has been widely demonstrated that the use of plant cell cultures can boost the comprehension of plant-environment interaction (Costa et al., 2010; Dubrovina et al., 2015; Larosa et al., 1987; Liu et al., 2013).

With this respect, our previous physiological studies have shown that potato cells adapted to water stress, in contrast to the cells subjected to shock, were able to grow in culture media supplemented with high concentrations of PEG and $\mathrm{NaCl}$. The cellular levels of abscisic acid (ABA), one of the major mediators of the signal of water deficit, were dramatically increased in cells subjected to shock, but remained unchanged in cell populations adapted to low osmotic potential. Finally, 


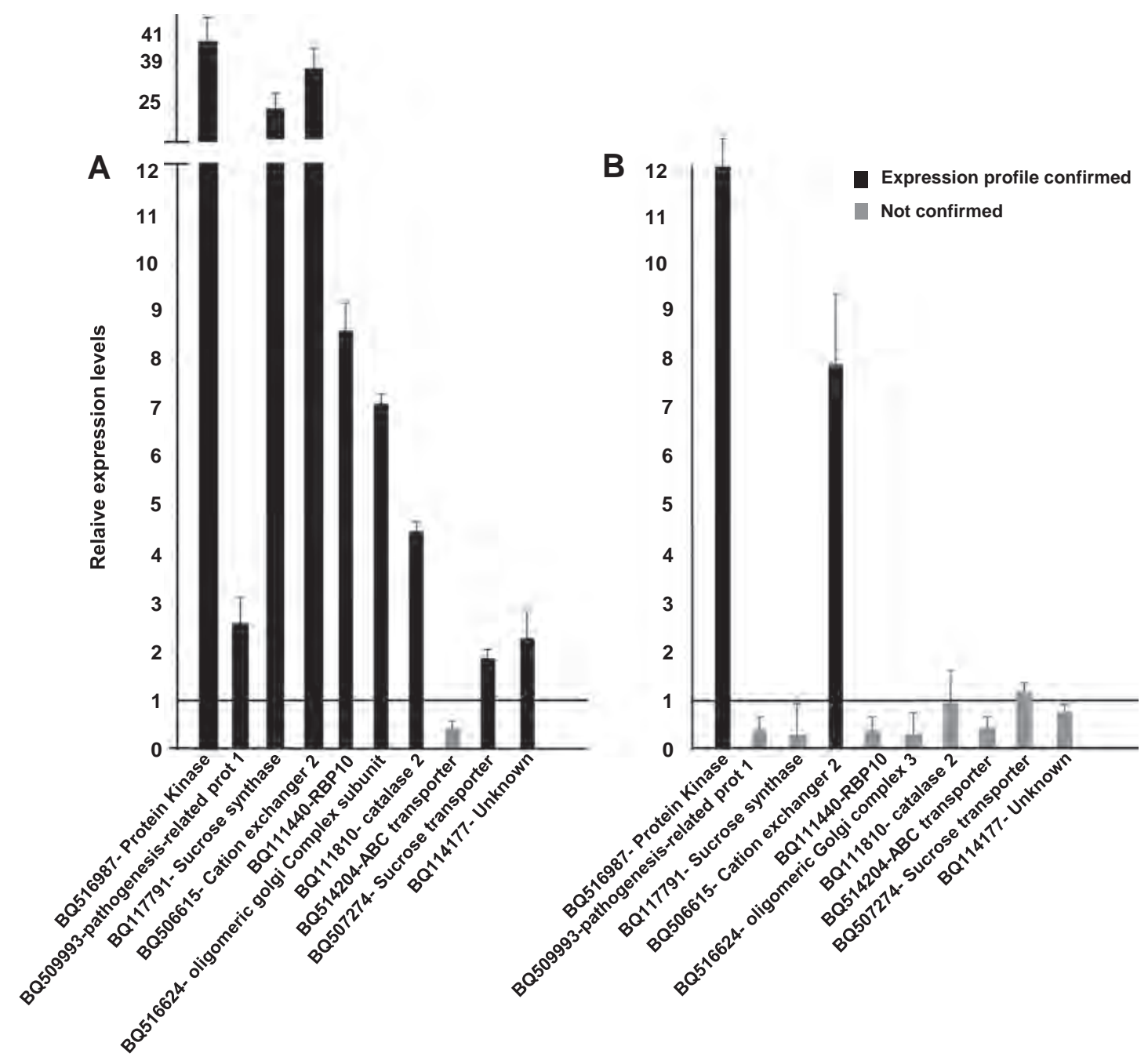

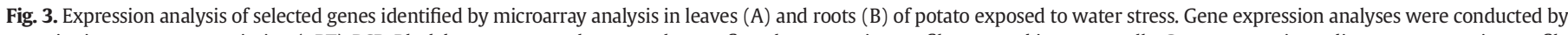

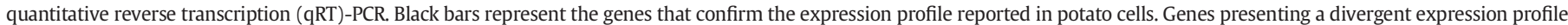
with respect to that detected in cells are reported in grey. Data are means \pm SD of three independent experiments.

adaptation to water stress restored the protein synthesis, dramatically inhibited in shock conditions (Leone et al., 1994b).

In order to identify genes involved in the two different aspects of the complex response mechanism to water stress and in particular, in the adaptation processes, we have characterized the transcriptome changes occurring in two potato cell populations subjected to abrupt or gradual water stress by microarray analysis, which still represents one of the most versatile large-scale analysis tool for many plant genomes, including potato (www.tigr.org). In recent years, data from microarray analyses allowed the identification and characterization of a large set of genes involved in mechanisms of plant response to different abiotic stresses (Bohnert et al., 2006), in particular to water deficit (Ambrosone et al., 2013; Ambrosone et al., 2015; Hazen et al., 2003; Kreps et al., 2002) confirming and emphasizing the complexity of the phenomenon.

\subsection{Network of genes involved in response to abrupt or gradual water stress}

The microarray analysis of RNA from potato cell populations showed a total of 1045 genes with altered expression profile compared to control conditions. In particular, 130 genes were found to be induced in shocked cells, 333 genes induced in adapted cells, 174 genes down-regulated in response to shock conditions and 408 genes down-regulated in the adaptation.
These data indicate that the molecular response, in terms of number of differentially regulated genes, is more complex in the adapted cell populations; in fact, a total of 740 genes showed an expression profile altered during adaptation to water deficit, in spite of about 300 genes induced or repressed in response to abrupt water stress conditions, suggesting that potato cells need to keep tuned a huge number of genes to adapt to gradual and prolonged water stress. Although it is extremely complex to compare transcriptomic data generated though different experimental systems and technology, the number of differentially expressed genes identified in our expression analyses is consistent to those observed in current literature (Gong et al., 2010; Iovieno et al., 2016; Vasquez-Robinet et al., 2008).

Moreover, the data analysis showed that only 9 genes are commonly regulated in gradually adapted and shocked cells. This clearly denotes that divergent mechanisms drive plant response to abrupt or gradual water deficit. Fascinatingly, abrupt imposition of an intense water stress activates transient gene expression programmes, which appear completely modified when potato cells experience a selective pressure determined by gradually adaptation to water limiting conditions. Thus, our data confirm, on one hand the general opinion that biological systems respond to environmental insults in a manner strictly dependent on the dose and duration of imposed stress and, on the other hand, shed light on molecular signatures of plant cellular adaptation to water deficit. In more details, genes overexpressed in the short 
term might code for important gene functions for a primary response to provide an initial protection against cell damages and to trigger stress signalling. Oppositely, genes activated in long-term response might have a direct role in the adaptation to the new needs dictated by the changing environment (Bray, 2004).

Overall, we found different sets of deregulated genes involved in transcriptional regulation, carbohydrate metabolism, protein synthesis, transport, detoxification signalling, osmolite production, hormone metabolism and nucleic acid metabolism. All these functional categories have been widely described through transcriptomic analyses as drought stress genes in many plant species (Alter et al., 2015; Borkotoky et al., 2013; Xue et al., 2008).

Finally, The microarray analysis also showed that most of the identified transcripts, from $35 \%$ to $50 \%$, has no similarity to experimentally characterized genes. Although at the present this class cannot contribute to argue stress response, in light of the upcoming functional genomics programs, these genes may serve as sources of interesting new gene functions not yet investigated.

Over the recent years great attention has been dedicated to dissect molecular pathways involved in potato water stress response. One of the first studies was carried out by Buell and colleagues (Rensink et al., 2005), who described the complex molecular response of potato plants to abiotic stresses such as cold, heat, salt and drought. Shortly, they demonstrated that low temperatures induce a deep change in the transcriptome with about 2584 genes with modified expression profile, while 1149 clones and 998 clones were induced or repressed in response to salt stress and high temperatures respectively.

Recently, interesting results were obtained from the study of the transcriptome of Andean potato species ( $S$. tuberosum ssp andigena) subjected to water stress. These studies showed that the drought tolerant accessions had a higher constitutive expression of genes involved in flavonoid biosynthesis (Watkinson et al., 2008) and in two subspecies of Andean potatoes (Sullu and SA2563) were found to be a greater accumulation of chaperone proteins such as heat shock proteins (HSP) and LEA (Schafleitner et al., 2007). Finally, a further analysis of expression in the Andean genotypes has suggested that the drought tolerance is related to the overexpression of plastidial proteins HSP and proteins with antioxidant activities and mithocondrial functions (Vasquez-Robinet et al., 2008). Despite this consistent literature on potato water stress response, our approach remains unique in the field, as it proposes a robust experimental setup conceived to identify molecular signatures inherently associated to abrupt water stress and gradual adaptation of $S$. tuberosum species. Our findings substantiate the hypothesis that different stress conditions evoke diverse molecular responses; besides comparative analysis, we identified a plethora of deregulated genes upon water deficit adaptation, providing new cues, which need further investigations. Below, we discussed deeper the major functional categories identified in this study, together with the role of key genes involved in potato cell response to water stress.

\subsection{Different sets of transcription drive plant response to abrupt and gradual water stress}

The expression of important transcription factors, as gigantea COL-1 (Costans like 1), a protein of the MYB family and SCARECROWN (SLC1), was induced in response to abrupt water stress. Interestingly, in Arabidopsis, gigantea COL-1 is activated in response to stress, in particular, its overexpression induces tolerance to oxidative stress conditions driving the expression of genes coding for superoxide dismutase (SOD) and for the ascorbate peroxidase enzyme (APX ) (Cao et al., 2006).

In adapted potato cells, transcription factors as TINY, WRKY40, STOP1, ARF22 and several proteins with zinc finger domains were overexpressed. TINY contains an APETALA2 domain and binds with high affinity to regulatory elements such as DRE (dehydrationresponsive element) and ERE (Ethylene-Responsive Element). The overexpression of this gene in Arabidopsis induces the activation of many transcripts containing the DRE and ERE elements, suggesting an important role in the mechanisms of cross-talking in response to stress of different nature (Sun et al., 2008). The up-regulation of WRKY40 gene in adapted cells is of particular interest. WRKY40 was up-regulated in Brassica and Arabidopsis in response to water limiting conditions and ABA treatment (Chen et al., 2010; Kayum et al., 2015). An interesting study showed that the expression of this gene was related to both abiotic and biotic stress responses, for which WRKY40 may be an additional important element in the plant defence mechanisms and in the cross-talk between different pathways (Ma and Bohnert, 2007).

Many transcription factors, known for their involvement in stress response, were down-regulated in cell cultures adapted to low water potentials. Among these, we found genes coding for WRKY54, WRKY 75, MYB factor, 2 TUBBY-like transcription factors.

The results are in agreement with previous findings identifying several transcription factors involved in plant responses to drought such as those belonging to the AP2/ERF families, basic leucine zipper (bZIP), NAC, MYB, MYC, WRKY and Cys2His2 zinc finger (Umezawa et al., 2006).

Overall, the results obtained indicate that a substantial remodelling of transcriptional factors was induced after water stress. It is possible to argue that while in shock conditions, the transcription factors may play a prominent role in sensing and transduction of external stimuli in order to activate early cellular processes to protect cell structures and functions; conversely, the transcription factors activated during long-term stress conditions may be part of an adaptive mechanism for metabolic and cellular processes reprogramming, thus ensuring cell survival in a changing environment.

\subsection{Abrupt water deficit transcriptionally impairs protein synthesis}

Protein synthesis is one of the most affected biochemical processes during stress exposure. In general, a strong inhibition of protein synthesis in response to water deficit has been reported in different plant species (Good and Zaplachinski, 1994).

We observed that many transcripts involved in protein synthesis were repressed in potato cells subjected to water shock; in particular, transcripts, coding for different ribosomal proteins (RPS3C, RPP3B, RPL10aB, RPL4D) and for signal-recognition-particle (SRP9) protein, were found to be negatively regulated. In contrast, only few genes involved in protein synthesis were found repressed in cell cultures adapted to low water potential. These data confirm and substantiate our previous proteomic investigation, which reported a strong reduction of protein synthesis in cells subjected to abrupt water stress conditions (Leone et al., 1994b). Taken together, these experimental evidences suggest that during osmotic shock conditions, potato cells genetically and biochemically inactivate key components of the translational machinery, slowing down the physiological protein turn over. Although not fully investigated, this behaviour could be a strategy to save cell energy and avoid the accumulation of unfolded, misfolded or damaged proteins, which in turn may fuel cell toxicity in unfavourable environment.

\subsection{Modulation of ethylene signalling network as a distinctive trait of potato cell adaptation}

Ethylene is an essential plant hormone that plays a fundamental role in many aspects of plant life cycle; its biosynthesis is finely regulated by endogenous signals during development as well as in response to biotic and abiotic environmental stimuli (Ecker, 1995). Anyway, its role in plant response and adaptation to osmotic stress is still debated and sometimes controversial (Tao et al., 2015).

Previously, we demonstrated that ethylene levels in potato adapted cells were six times higher than the control cells, while no significant 
changes in ethylene levels were detected in cells subjected to shock conditions (Scaramagli et al., 2000). These data pointed out that ethylene may have a prominent role during adaption to water deficit in cell culture. Interestingly, most of genes involved in ethylene biosynthesis and signalling were deregulated in potato adapted cells. Basically, we observed the overexpression of central players of ethylene signalling such as S-adenosylmethionine synthetase (SAM2), aminocyclopropane-1-carboxylic acid synthase (ACS or ACC synthase), and the ethylene receptor, ETHYLENE INSENSITIVE4 (EIN4) in potato adapted cells. Among these genes, ACC synthase has already been linked to drought stress (Alter et al., 2015).

Roughly speaking, SAM2 catalyzes the formation of Sadenosylmethionine (S-AdoMet) which represents the major precursor of ethylene biosynthesis. Then, ACS catalyzes the first biosynthetic reaction of ethylene pathway by converting S-adenosylmethionine (SAdoMet) in 1-aminocyclopropane-1-carboxylic acid (ACC); then the ethylene synthase proceeds by oxidation of the ACC through an ACC oxidase (ACO). Overall, the ethylene levels are controlled by negative feedback mechanisms that act on the major biosynthetic reactions (Wang et al., 2002).

In our cellular system, the up-regulation of both SAM2 and ACS transcripts indicate that adapted cells actively sustain the biosynthesis of ethylene and its precursors over long-term osmotic conditions. In addition, the suppression of the ACO enzyme transcription could represent an attempt to limit the hormone production by a negative feedback regulation in presence of high ethylene concentrations. We also observed the overexpression of EIN4, one of the ethylene receptors (Hua et al., 1998), which confirms high specific signalling activity.

Finally, among differentially expressed transcripts in potato adapted cells we noticed an impressive number of genes involved in defence to pathogens, which may correlate with the ethylene biological activity to prompt biotic cell response (Ecker and Davis, 1987). Interestingly, some of these genes have already been linked to abiotic stress responses such as mildew resistance locus (MLO), disease resistance protein 2 (RGA 2) and syntaxin 121 (SYP121) (Hachez et al., 2014; Melloul et al., 2014; Piffanelli et al., 2002).

To conclude, beyond the well consolidate role of ethylene in the crosstalk of biotic and abiotic signalling, these data indicate that this hormone may serve as an important stress modulator, especially during gradual adaptation to water limiting conditions. As consequence, future experiments need to further address the underestimated role of ethylene in plant long-term water stress adaptation.

\subsection{Moving from the in vitro to the in vivo water deficit response: An interesting lesson}

To assess if in vitro outcomes might provide useful cues for in vivo water stress response, we profiled the expression of 10 selected stress responsive genes identified by potato microarray analysis in potato plants under slow soil drying conditions. The expression study showed that 9 out of 10 of the analyzed transcripts in cells was over-expressed in leaves collected from plants exposed to water deficit conditions; some transcripts also presented an induction level much higher in the plant tissue that in cells, suggesting a concrete involvement of these genes in the plant response. Among the major overexpressed genes in the leaves, we identified transcripts encoding a protein kinase, a sucrose synthase (sus4), an ion channel (cax2), a catalase (cat2). The sucrose synthase (sus4) is a key enzyme in the metabolism of sugars; it catalyzes the reversible conversion of sucrose into UDP-glucose and fructose. $S$. tuberosum sus 4 isoform is reported to be constitutively expressed at high levels in tubers, buds and meristems (Fu et al., 1995). Sus4 gene expression is triggered by anoxia, while it seems not affected by water stress in Arabidopsis (Baud et al., 2004).

Anyway, drought induces the expression of two sucrose synthase isoforms, SUS3 and SUS4, in potato leaves (Kondrák et al., 2012). Interestingly, this gene turned out to be associated with nucleic acid break down during leaf senescence in cucumber and rapeseed (BuchananWollaston, 1997). Anyway, increased concentrations of soluble sugars have often been reported under osmotic stress such as drought and salinity, possibly to avoid or recover from embolism (Secchi and Zwieniecki, 2016). Although not deeply investigated in this work, the huge overexpression of sus4 may regulate osmotic homeostasis of cells as well as boost sugar mobilization in order to reduce the risk of embolisms in potato plants.

The catalase 2 (cat2) plays a key role in cell detoxification from reactive oxygen species (ROS); in particular, it catalyzes the conversion of hydrogen peroxide into water and oxygen (Scandalios, 1997). Overexpression of cat2 in potato cells and plants represents a clear attempt to reduce the excess of ROS radicals generated from water deficit.

In roots of plants grown under water deficit, only two genes (a kinase and the cation exchanger 2, $\operatorname{cax} 2$ ) were induced. It is possible to hypothesize that these two genes serve as members of the general protective mechanisms activated in response to stress, intervening in a ubiquitous manner in water limiting conditions.

In conclusion, we successful used a potato cell system to identify important genes involved in plant stress response to short and long-term water deficit. Transcriptome profiling confirmed that adaptation to water limiting conditions requires an impressive modulation of gene expression, much more complex than biological response to short term stress.

Although this study did not focus on water stress response in adult plants, we proved that genes identified by microarray analysis in response to stress were differentially expressed in vivo as well. Therefore, beyond the type of stress experienced by potato plants, our results provide a helpful list of candidate genes to be considered for further functional analysis in order to establish their roles in plant adaptation/ tolerance. As final remark, epigenetic mechanisms may establish large changes in gene expression patterns, guiding gradual plant adaptation over a changing environment (Kinoshita and Seki, 2014; Mirouze and Paszkowski, 2011), therefore it remains of intriguing interest to investigate the epigenetic modifications that contribute to reshape the transcriptional landscape of potato cells in our experimental system.

Supplementary data to this article can be found online at doi:10. 1016/j.gene.2016.10.024.

\section{Conflict of interest}

The authors declare that they have no competing interests.

\section{Aknowledgements}

This work was funded by a grant from the Italian Ministry of Research (MIUR, PON02_00395_3215002 'GenHort'). We express our thanks to Gaetano Guarino, Rosario Nocerino and Roberta Nurcato for excellent technical assistance.

\section{References}

Alter, S., Bader, K.C., Spannagl, M., Wang, Y., Bauer, E., Scho, C.C., Mayer, K.F.X., 2015 DroughtDB: an expert-curated compilation of plant drought stress genes and their homologs in nine species Database 1-7 http://dx.doi.org/10.1093/database/bav046.

Ambrosone, A., Costa, A., Martinelli, M., Massarelli, I., De Simone, V., Grillo, S., Leone, A. 2011. Differential gene regulation in potato cells and plants upon abrupt or gradual exposure to water stress. Acta Physiol. Plant. 33, 1157-1171.

Ambrosone, A., Costa, A., Leone, A., Grillo, S., 2012. Beyond transcription: RNA-binding proteins as emerging regulators of plant response to environmental constraints. Plant Sci. 182, 12-18.

Ambrosone, A., Di Giacomo, M., Leone, A., Grillo, S., Costa, A., 2013. Identification of early induced genes upon water deficit in potato cell cultures by cDNA-AFLP. J. Plant Res. 126, 169-178.

Ambrosone, A., Batelli, G., Nurcato, R., Aurilia, V., Punzo, P., Bangarusamy, D.K., Ruberti, I., Sassi, M., Leone, A., Costa, A., Grillo, S., 2015. The arabidopsis RNA-binding protein AtRGGA regulates tolerance to salt and drought stress plant physiology. 168 pp. 292-306.

Araus, J.L., Slafer, G.A., Reynolds, M.P., Royo, C., 2002. Plant breeding and drought in C3 cereals: what should we breed for? Ann. Bot. 89, 925-940. 
Baud, S., Vaultier, M.N., Rochat, C., 2004. Structure and expression profile of the sucrose synthase multigene family in Arabidopsis. J. Exp. Bot. 55 (396), 397-409.

Blum, A., 2005. Drought resistance, water-use efficiency, and yield potential-are they compatible, dissonant, or mutually exclusive? Aust. J. Agric. Res. 56, 1159-1168.

Bohnert, H.J., Qingqiu, G., Li, P., Ma, S., 2006. Unraveling abiotic stress tolerance mechanisms - getting genomics going. Curr. Opin. Plant Biol. 9, 180-188.

Borkotoky, S., Saravanan, V., Jaiswal, A., Das, B., Selvaraj, S., Murali, A., Lakshmi, P.T.V., 2013. The Arabidopsis stress responsive gene database. Int. J. Plant Genomics 2013, 1-4.

Bray, E.A., 2002. Classification of genes differentially expressed during water-deficit stress in Arabidopsis thaliana: an analysis using microarray and differential expression data. Ann. Bot. 89, 803-811.

Bray, E.A., 2004. Genes commonly regulated by water-deficit stress in Arabidopsis thaliana. J. Exp. Bot. 55, 2331-2341.

Buchanan-Wollaston, V., 1997. The molecular biology of leaf senescence. J. Exp. Bot. 48 (2), 181-199.

Cao, S.Q., Ye, M., Huang, Q., Zhang, R.X., 2006. A role for SPINDLY gene in the regulation of oxidative stress response in Arabidopsis. Russ. J. Plant Physiol. 53, 1021-4437.

Chen, H., Lai, Z., Shi, J., Xiao, Y., Chen, Z., Xu, X., 2010. Roles of arabidopsis WRKY18, WRKY40 and WRKY60 transcription factors in plant responses to abscisic acid and abiotic stress. BMC Plant Biol. 10, 281.

Chomeczynski, P., Sacchi, N., 1987. Single-step method of RNA isolation by acid guanidinium thiocyanate phenol chloroform extraction. Anal. Biochem. 162, 156-159.

Claeys, H., Inzé, D., 2013. The agony of choice: how plants balance growth and survival under water-limiting conditions. Plant Physiol. 162, 1768-1779.

Claeys, H., Van Landeghem, S., Dubois, M., Maleux, K., Inzé, D., 2014. What is stress? Doseresponse effects in commonly used in vitro stress assays. Plant Physiol. 165, 519-527.

Clauw, P., Coppens, F., De Beuf, K., Dhondt, S., Van Daele, T., Maleux, K., Storme, V. Clement, L., Gonzalez, N., Inzé, D., 2015. Leaf responses to mild drought stress in natural variants of Arabidopsis thaliana. Plant Physiol. 167 (3), 800-816.

Cleveland, W., Devlin, S., 1988. Locally weighted linear regression: an approach to regression analysis by local fitting. J. Am. Stat. Assoc. 83, 596-609.

Costa, A., Di Giacomo, M., Massarelli, M., De Palma, M., Leone, A., Grillo, M.S., 2010. Isolation, characterization and expression of an elongation factor $1 \alpha$ gene in potato (Solanum tuberosum) cell cultures. Plant Biol. 144 (3), 618-625.

Deblonde, P.M.K., Ledent, J.F., 2001. Effects of moderate drought conditions on green leaf number, stem height, leaf length and tuber yield of potato cultivars. Eur. J. Agron. 14 $31-41$.

Dobbin, K., Shih, J.H., Simon, R., 2003. Questions and answers on design of dual-label microarrays for identifying differentially expressed genes. J. Natl. Cancer Inst. 95, 1362-1369.

Dubrovina, A.S., Kiselev, K.V., Khristenko, V.S., Aleynova, O.A., 2015. VaCPK20, a calciumdependent protein kinase gene of wild grapevine Vitis amurensis Rupr., mediates cold and drought stress tolerance. J. Plant Physiol. 185, 1-12.

Ecker, J.R., 1995. The ethylene signal transduction pathway in plants. Science 268 (5211), 667-675.

Ecker, J.R., Davis, R.W., 1987. Plant defense genes are regulated by ethylene. Proc. Natl. Acad. Sci. U. S. A. 84 (15), 5202-5206.

Fu, H., Kim, S.Y., Park, W.D., 1995. High-level tuber expression and sucrose inducibility of a potato Sus 4 sucrose synthase gene require $5^{\prime}$ and $3^{\prime}$ flanking sequences and the leader intron. Plant Cell 7, 1387-1394.

Gong, P., Zhang, J., Li, H., Yang, C., Zhang, C., Zhang, X., Khurram, Z., Zhang, Y., Wang, T., Fei, Z., Ye, Z., 2010. Transcriptional profiles of drought-responsive genes in modulating transcription signal transduction, and biochemical pathways in tomato. J. Exp. Bot. 61 (13), 3563-3575.

Good, A.G., Zaplachinski, S.T., 1994. The effects of drought stress on free amino acid accumulation and protein synthesis in Brassica napus. Physiol. Plant. 90, 9-14.

Gremme, G., Brendel, V., Sparks, M.E., Kurtz, S., 2005. Engineering a software tool for gene structure prediction in higher organisms. Inf. Softw. Technol. 47 (15), 965-978.

Grillo, S., Costa, A., Tucci, M., Consiglio, F., Amatruda, M.R., Leone, A., 1996. Regulation of gene expression during cellular adaptation to water stress. Physical Stresses in Plants. Genes and their Products for Tolerance (Eds S. Grillo \& A. Leone). Springer-Verlag, Heidelberg, pp. 163-169.

Gullì, M., Salvatori, E., Fusaro, L., Pellacani, C., Manes, F., Marmiroli, N., 2015. Comparison of drought stress response and gene expression between a GM maize variety and a near-isogenic non-GM variety. PLoS One 10 (2), e0117073.

Hachez, C., Laloux, T., Reinhardt, T., Cavez, D., Degand, H., Grefen, C., De Rycke, R., Inzè, D., Blatt, M.R., Russinova, E., Chaumont, F., 2014. Arabidopsis SNAREs SYP61 and SYP121 coordinate the trafficking of plasma membrane aquaporin PIP2; 7 to modulate the cell membrane water permeability. Plant Cell 26 (7), 3132-3147.

Hazen, S.P., Wu, Y., Kreps, J.A., 2003. Gene expression profiling of plant responses to abiotic stress. Funct. Integr. Genomics 3, 105-111.

Hirsch, C.D., Hamilton, J.P., Childs, K.L., Cepela, J., Crisovan, E., Vaillancourt, B., Hirsch, C.N., Habermann, M., Neal, B., Buell, C.R., 2014. Spud DB: a resource for mining sequences, genotypes, and phenotypes to accelerate potato breeding. The Plant Genome 7 (1). http://dx.doi.org/10.3835/plantgenome2013.12.0042.

Hua, J., Sakaia, H., Nourizadehb, S., Chenc, Q.G., Bleeckerc, A.B., Eckerb, J.R., Meyerowitza, E.M., 1998. EIN4 and ERS2 are members of the putative ethylene receptor gene family in Arabidopsis. Plant Cell 10 (8), 1321-1332.

Iovieno, P., Punzo, P., Guida, G., Mistretta, C., Van Oosten, M.J., Nurcato, R., Bostan, H., Colantuono, C., Costa, A., Bagnaresi, P., Chiusano, M.L., Albrizio, R., Giorio, P., Batelli, G., Grillo, S., 2016. Transcriptomic changes drive physiological responses to progressive drought stress and rehydration in tomato. Front. Plant Sci. 7, 371.
Kayum, M.A., Jung, H.J., Park, J.I., Ahmed, N.U., Saha, G., Yang, T.J., Nou, I.S., 2015. Identification and expression analysis of WRKY family genes under biotic and abiotic stresses in Brassica rapa. Mol. Gen. Genomics. 290, 79-95.

Kinoshita, T., Seki, M., 2014. Epigenetic memory for stress response and adaptation in plants. Plant Cell Physiol. 55 (11), 1859-1863.

Kondrák, M., Marincs, F., Antal, F., Juhász, Z., Bánfalvi, Z., 2012. Effects of yeast trehalose-6phosphate synthase 1 on gene expression and carbohydrate contents of potato leaves under drought stress conditions. BMC Plant Biol. 12, 74.

Kreps, J.A., Wu, Y., Chang, H.S., Zhu, T., Wang, X., Harper, J.F., 2002. Transcriptome changes for Arabidopsis in response to salt, osmotic and cold stress. Plant Physiol. 230, 2129-2141.

Larosa, P.C., Hasegawa, P.M., Rhodes, D., Clithero, J.M., Watad, A.E., Bressan, R.A., 1987. Abscisic acid stimulated osmotic adjustment and its involvement in adaptation of tobacco cells to NaCl. Plant Physiol. 85 (1), 174-181.

Legay, S., Lamoureux, D., Hausman, J.F., Hoffmann, L., Evers, D., 2009. Monitoring gene expression of potato under salinity using cDNA microarrays. Plant Cell Rep. 28 (12), 1799-1816.

Leone, A., Costa, A., Tucci, M., Grillo, S., 1994a. Adaptation versus shock response to PEGinduced low water potential in cultured potato cells. Physiol. Plant. 92, 21-30.

Leone, A., Costa, A., Tucci, M., Grillo, S., 1994b. Comparative analysis of short- and longterm changes in gene expression caused by low potential in potato (Solanum tuberosum) cell-suspension cultures. Plant Physiol. 106, 703-712.

Leone, A., Costa, A., Grillo, S., Tucci, M., Horvarth, I., Vigh, L., 1996. Acclimation to low water potential determines changes in plasma membrane fatty acid composition and fluidity in potato cells. Plant Cell Environ. 19, 1103-1109.

Leone, A., Costa, A., Consiglio, F., Massarelli, I., Dragonetti, E., De Palma, M., Grillo, S., 1999. Tolerance to abiotic stresses in potato plants: a molecular approach. Potato Res. 42, 333-351.

Li, C., Nong, O., Solanki, M.K., Liang, Q., Xie, J., Liu, X., Li, Y., Wang, W., Yang, L., Li, Y., 2016. Differential expression profiles and pathways of genes in sugarcane leaf at elongation stage in response to drought stress. Sci. Rep. 6, 25698.

Liu, D., Ford, K.L., Roessner, U., Natera, S., Cassin, A.M., Patterson, J.H., Bacic, A., 2013. Rice suspension cultured cells are evaluated as a model system to study salt responsive networks in plants using a combined proteomic and metabolomic profiling approach. Proteomics 13, 2046-2062.

Livak, K.J., Schmittgen, T.D., 2001. Analysis of relative gene expression data using realtime quantitative PCR and the 2(-Delta Delta C(T)) method. Methods 25, 402-408.

Ma, S., Bohnert, H.J., 2007. Integration of Arabidopsis thaliana stress-related transcript profiles, promoter structures, and cell-specific expression. Genome Biol. 8, R49.

Melloul, M., Iraqi, D., El Alaoui, M., Erba, G., Alaoui, S., Ibriz, M., Elfahime, E., 2014. Identification of differentially expressed genes by cDNA-AFLP technique in response to drought stress in Triticum durum. Food Technol. Biotechnol. 52 (4), 479-488.

Mirouze, M., Paszkowski, J., 2011. Epigenetic contribution to stress adaptation in plants. Curr. Opin. Plant Biol. 14 (3), 267-274.

Piffanelli, P., Zhou, F., Casais, C., Orme, J., Jarosch, B., Schaffrath, U., Collins, N.C., Panstruga, R., Shulze-Lefert, P., 2002. The barley MLO modulator of defense and cell death is responsive to biotic and abiotic stress stimuli. Plant Physiol. 129 (3), 1076-1085.

Rensink, W.A., Iobst, S., Hart, A., Stegalkina, S., Liu, J., Buell, C.R., 2005. Gene expression profiling of potato responses to cold, heat, and salt stress. Funct Int Genomics 5, 201-207.

Roche, J., Hewezi, T., Bouniols, A., Gentzbittel, L., 2008. Transcriptional profiles of primary metabolism and signal transduction-related genes in response to water stress in field-grown sunflower genotypes using a thematic cDNA microarray. Planta 226 (3), 601-617.

Ruepp, A., Zollner, A., Maier, D., Albermann, K., Hani, J., Mokrejs, M., Tetko, I., Güldener, U., Mannhaupt, G., Münsterkötter, M., Mewes, H.W., 2004. The FunCat, a functional annotation scheme for systematic classification of proteins from whole genomes. Nucleic Acids Res. 32, 5539-5545.

Scandalios, J.G., 1997. Oxidative stress and defense mechanisms in plants: introduction. Free Radic. Biol. Med. 23, 471-472.

Scaramagli, S., Biondi, S., Leone, A., Grillo, S., Torrigiani, P., 2000. Acclimation to low water potential in potato cell suspension cultures leads to changes in putrescine metabolism. Plant Physiol. Biochem. 38, 345-351.

Schafleitner, R., Gutierrez Rosales, R.O., Gaudin, A Alvarado Aliaga, CA Martinez, G.N. Tincopa Marca, L.R., Bolivar, L.A., Delgado, F.M., Simon, R., Bonierbale, M., 2007. Capturing candidate drought tolerance traits in two native Andean potato clones by transcription profiling of field grown plants under water stress. Plant Physiol. Biochem. 45, 673-690.

Secchi, F., Zwieniecki, M.A., 2016. Accumulation of sugars in the xylem apoplast observed under water stress conditions is controlled by xylem pH. Plant Cell Environ. http://dx. doi.org/10.1111/pce.12767.

Seki, M., Narusaka, M., Ishida, J., Nanjo, T., Fujita, M., Oono, Y., Kamiya, A., Nakajima, M., Enju, A., Sakurai, T., Satou, M., Akiyama, K., Taji, T., Yamaguchi-Shinozaki, K., Carninci, P., Kawai, J., Hayashizaki, Y., Shinozaki, K., 2002. Monitoring the expression profiles of 7000 Arabidopsis genes under drought, cold and high-salinity stresses using a full-length cDNA microarray. Plant J. 31, 279-292.

Seki, M., Kamei, A., Yamaguchi-Shinozaki, K., Shinozaki, K., 2003. Molecular responses to drought, salinity and frost: common and different paths for plant protection. Curr. Opin. Biotechnol. 14, 194-199.

Shinozaki, K., Yamaguchi-Shinozaki, K., Seki, M., 2003. Regulatory network of gene expression in the drought and cold stress responses. Curr. Opin. Plant Biol. 6, 410-417.

Sun, S., Yu, J.P., Chen, F., Zhao, T.J., Fang, X.H., Li, Y.Q., Sui, S.F., 2008. TINY, a dehydrationresponsive element (DRE)-binding protein-like transcription factor connecting the DRE- and ethylene-responsive element-mediated signaling pathways in Arabidopsis. J. Biol. Chem. 283, 6261-6271. 
Takahashi, S., Seki, M., Ishida, J., Satou, M., Sakurai, T., Narusaka, M., Kamiya, A., Nakajima, M., Enju, A., Akiyama, K., Yamaguchi-Shinozaki, K., Shinozaki, K., 2004. Monitoring the expression profiles of genes induced by hyperosmotic, high salinity, and oxidative stress and abscisic acid treatment in Arabidopsis cell culture using a full-length cDNA microarray. Plant Mol. Biol. 56, 29-55.

Tao, J.J., Chen, H.W., Ma, B., Zhang, W.K., Chen, S.Y., Zhang, J.S., 2015. The role of ethylene in plants under salinity stress. Front. Plant Sci. 6, 1059.

Tatusova, T.A., Madden, T.L., 1999. BLAST 2 sequences, a new tool for comparing protein and nucleotide sequences. FEMS Microbiol. Lett. 174 (2), 247-250.

The Potato Genome Sequencing Consortium, 2011. Genome sequence and analysis of the tuber crop potato. Nature 475, 189-195.

Umezawa, T., Fujita, M., Fujita, Y., Yamaguchi-Shinozaki, K., Shinozaki, K., 2006. Engineering drought tolerance in plants: discovering and tailoring genes to unlock the future. Curr. Opin. Biotechnol. 17, 113-122.

Urano, K., Kurihara, Y., Seki, M., Shinozaki, K., 2010. ‘Omics’ analyses of regulatory networks in plant abiotic stress responses. Curr. Opin. Plant Biol. 13, 132-138.

Vasquez-Robinet, C., Mane, S.P., Ulanov, A.V., Watkinson, J.I., Stromberg, V.K., De Koeyer, D., Schafleitner, R., Willmot, D.B., Bonierbale, M., Bohnert, H.J., Grene, R., 2008.
Physiological and molecular adaptations to drought in Andean potato genotypes. J. Exp. Bot. 59 (8), 2109-2123.

Wang, K.L., Li, H., Ecker, J.R., 2002. Ethylene biosynthesis and signaling networks. Plant Cell 14, 131-151.

Watkinson, J.I., Hendricks, L., Sioson, A.A., Heath, L.S., Bohnert, H.J., Grene, R., 2008. Tuber development phenotypes in adapted and acclimated, drought-stressed Solanum tuberosum ssp. andigena have distinct expression profiles of genes associated with carbon metabolism. Plant Physiol. Biochem. 46 (1), 34-45.

Weisz, R., Kaminski, J., Smilowitz, Z., 1994. Water-deficit effects on potato leaf growth and transpiration: utilizing fraction extractable soil-water for comparison with other crops. Am. Potato J. 71, 829-840.

Xue, G.P., McIntyre, C., Glassop, L., Shorter, R., 2008. Use of expression analysis to dissect alterations in carbohydrate metabolism in wheat leaves during drought stress. Plant Mol. Biol. 67, 197-214.

Yamaguchi-Shinozaki, K., Shinozaki, K., 2005. Organization of cis-acting regulatory elements in osmotic- and cold-stress-responsive promoters. Trends Plant Sci. 10 (2), 88-94.

Zhu, J.K., 2001. Plant salt tolerance. Trends Plant Sci. 6, 66-71. 